\title{
An Adaptive Image Segmentation Method Based on the Level Set
}

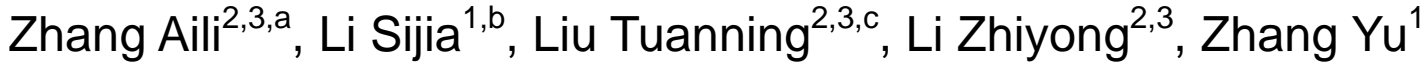 \\ ${ }^{1}$ College of Physics and Electronic Engineering, Henan Normal University, Xinxiang 453007, China \\ ${ }^{2}$ College of Computer and Information Engineering, Henan Normal University, Xinxiang 453007, \\ China \\ ${ }^{3}$ Engineering Lab of Henan Province for Intelligence Business \& Internet of Things, Xinxiang \\ 453007, China \\ azal5148@126.com, blisijia1234@126.com, 'liutuan123@126.com
}

Keywords: Uneven information; Global segmentation; Fitting Information; Image segmentation

\begin{abstract}
An adaptive gray value of image segmentation method aimed at uneven gray-level image segmentation was proposed. The innovative method combined target image with the gray value of background information according to level set theory with the adaptive linear fitting of global and local information. And then, image segmentation was conducted to avoid being trapped in the local optimum. The experimental results showed that the proposed method could resist noise effectively with the capacity of adaptive image uneven gray level information. As a result, The global optimal image segmentation was obtained. Theoretical analysis suggested that the proposed method not only can be used to cut up the real image but also can be well applied to MRI segmentation.
\end{abstract}

\section{Introduction}

As the main research area in computer vision processing, image segmentation has been emerging in recent years. However, changes in illuminance unevenness space and defect image forming apparatus result in image gradation unevenness ${ }^{[1-4]}$, which is such a big problem in image segmentation that the traditional image segmentation methods can not handle grayscale images. In the literature[5.6], C.M. Li proposed LBF model, able to handle uneven gray image segmentation effectively with the local information of images. In addition, the model could also be used in MRI segmentation $^{[7,8]}$. Later, C.M. Li put forward an improved LBF model that could make good use of local image intensity inhomogeneity information to conduct image segmentation. K.zhang pointed out the shortcoming of this method it is easy to get trapped in local minimum due to distance regularization constraints. Aimed at the problem, this article based on C.M. Li's model, combined global gray level information with that of local to achieve adaptive fit of the above information, which in turn not only could operate the global image segmentation to avoid falling into a very small value, but also had good noise robustness. On one hand, the method proposed in this article could be used for real image segmentation, on the other hand, it was also able to handle MRI segmentation.

\section{Improved LBF model}

LBF model uses information uneven gray image segmentation to achieve a better one. Based on this, an improved method of LBF model is proposed by C.M.Li, which regards the nonuniform grayscale image component as a table, taking into account the large number of physical factors of the image. The image is represented by the formula (1).

$$
I=b J+n
$$

$I$ represents the actual image, $b$ indicating uneven gray image information, $n$ with indication additive noise. Real image $J$ represents an inherent property of the imaged object, assuming $J$ is a piecewise constant. Energy level set-based pan-function model ${ }^{[9]}$ is represented by the formula (2). 


$$
F(\phi, C, b)=E(\phi, C, b)+v \ell(\phi)+u \Re(\phi)
$$

Wherein, the regulation terms $\ell(\phi)$ and $\mathfrak{R}(\phi)$ are defined as:

$$
\left\{\begin{array}{l}
\ell(\phi)=\int|\nabla H(\phi)| d x \\
\mathfrak{R}_{p}=\int p(\nabla \phi) d x
\end{array}\right.
$$

Wherein, $\ell(\phi)$ representing the length of the zero level set contour is distance regulation.

$$
E(\phi, C, b)=\int_{\Omega} \int_{\text {inside }(C)} K_{\sigma}(y-x)\left|I(x)-b(y) c_{1}\right|^{2} H(\phi) d x d y+\int_{\Omega} \int_{\text {outside }(C)} K_{\sigma}(y-x)\left|I(x)-b(y) c_{2}\right|^{2} d x d y
$$

Wherein, $I(x)$ is for the original input image, $K_{\sigma}$ as the standard deviation is equal to the Gaussian kernel $\sigma, \mathrm{c} 1$ and c2 are the average gray values within and outside the contour region.

Hay function[10] is expressed as:

$$
H(z)=\frac{1}{2}\left[1+\frac{2}{\pi} \arctan \left(\frac{z}{\varepsilon}\right)\right]
$$

Differential $\mathrm{H}$ are indicated:

$$
\delta_{\varepsilon}(z)=H^{\prime}(z)=\frac{1}{\pi} \frac{\varepsilon}{\varepsilon^{2}+z^{2}}, z \in R
$$

The above formula shows that $\varepsilon$ will affect the profile parameter $\delta_{\varepsilon}(z)$. Too large $\varepsilon$ will result in excessive contour, thereby increasing the capture region and the contour segmentation accuracy decreases.

Using the level set method, Lipschitz function represented $C \subset R$ by the zero level set, the level set function is represented as $\phi, \phi: \Omega \subset R$, to derivative the contour C, get the gradient of $\phi$ [11].

$$
\frac{\partial \phi}{\partial t}=-\delta_{\varepsilon}(\phi)\left(\lambda_{1} e_{1}-\lambda_{2} e_{2}\right)
$$

In order to make a smooth evolution of the level set function, increasing the distance regularization term, and Used Euler length term regularizing level set to zero outline [12], Finally, the evolution formula of function is:

$$
\frac{\partial \phi}{\partial t}=u \operatorname{div}\left(d_{p} \frac{\nabla \phi}{|\nabla \phi|}\right)+v \delta_{\varepsilon}(\phi) \operatorname{div}\left(\frac{\nabla \phi}{|\nabla \phi|}\right)-\delta_{\varepsilon}(\phi)\left(\lambda_{1} e_{1}-\lambda_{2} e_{2}\right)
$$

Wherein, coefficient $\lambda_{1}, \lambda_{2}$ represents the area inside and outside contour integral right weight, general provisions $\lambda_{1}=\lambda_{2}=1$, the definition $e_{1}, e_{2}$ of the formula (9), and regulation,

$$
\begin{gathered}
d_{p}=\frac{p^{\prime}(s)}{s}, \quad p(s)=(1 / 2)(s-1)^{2} \\
\left\{\begin{aligned}
e_{1} & =\int_{\Omega} K_{\sigma}(y-x)\left|I(x)-b(y) c_{1}\right|^{2} d y \\
& =I^{2} 1_{K}-2 c_{1} I\left(b^{*} K\right)+c_{1}^{2}\left(b^{2} * K\right) \\
e_{2} & =\int_{\Omega} K_{\sigma}(y-x)\left|I(x)-b(y) c_{2}\right|^{2} d y \\
& =I^{2} 1_{K}-2 c_{2} I\left(b^{*} K\right)+c_{2}^{2}\left(b^{2} * K\right)
\end{aligned}\right.
\end{gathered}
$$

$1_{K}$ is defined as $1_{K}(x)=\int K_{\sigma}(y-x) d y$, in addition to any other one of the regions of the image area is constant 1.

Uneven grayscale image information $b$ as an integral part of the image $I$ information, the effective use of the image gray uneven information on the time and cost of segmentation algorithm are superior to CV model[13] and LBF model. However, due to the simulation, results show that the algorithm considers only locally uneven grayscale image information, the global image segmentation results are unsatisfactory.

1. Image to fit the local and global information

The following information will be gray outline of the image to fit inside and outside, by taking into account local and global information for image segmentation. In the image evolution, considering the gray information inside and outside the contour $\mathrm{C}$, equation (9) becomes: 


$$
\begin{array}{r}
\left\{\begin{array}{r}
e_{1}=c_{1}+\omega_{1} \cdot\left(I^{2} 1_{K}-2 c_{1} I\left(b^{*} K\right)+c_{1}^{2}\left(b^{2} * K\right)\right) \\
e_{2}=c_{2}+\omega_{2} \cdot\left(I^{2} 1_{K}-2 c_{2} I(b * K)+c_{2}^{2}\left(b^{2} K\right)\right)
\end{array}\right. \\
\text { Wherein, }\left\{\begin{array}{l}
\omega_{1}=\frac{c_{1}}{c_{1}+c_{2}} \\
\omega_{2}=\frac{c_{2}}{c_{1}+c_{2}}
\end{array}\right.
\end{array}
$$

Wherein, $c_{1}, c_{2}$ respectively represent the average gray scale information within and outside the contour $C$. In order to achieve the minimum energy $\mathrm{F}$, at every iteration, the updated level set $\phi$, the contour $C$, the deviation area $b$, the energy $F(\phi, C, b)$ is minimized, resulting in deviation corrected image. Update algorithm is as follows:

Step 1: fix $C$ and $b$, the function $F(\phi, C, b)$ is about the $\phi$ function. By the formula (2), the function $F(\phi, C, b)$ can be an minimized energy function, in this process, the constants $c_{1}, c_{2}$, and the bias term by the formula (4) is updated, wherein, $e_{1}$ and $e_{2}$ are for the formula (10).

Step2: fix $\phi$ and $b$, function $E(\phi, C, b)$ minimizing the energy with respect to $C$ is updated as:

$$
\left\{\begin{array}{l}
c_{1}=\frac{\int\left(b^{*} K_{\sigma}\right) I H(\phi) d y}{\int\left(b^{2} * K_{\sigma}\right) H(\phi) d y} \\
c_{2}=\frac{\int\left(b^{*} K_{\sigma}\right) I(1-H(\phi)) d y}{\int\left(b^{2} * K_{\sigma}\right)(1-H(\phi)) d y}
\end{array}\right.
$$

Step3: fix $\phi$ and $C$, function $E(\phi, C, b)$ minimizing the energy with respect to b is updated as:

$$
\hat{b}=\frac{\left(I J^{(1)}\right) * K_{\sigma}}{J^{(2)} * K_{\sigma}}
$$

Wherein $J^{(1)}=\sum_{i=1}^{N} c_{i} u_{i}, \quad J^{(2)}=\sum_{i=1}^{N} c_{i}^{2} u_{i}$

Experimental results and analysis

The proposed combination of global and local information gray uneven level set evolution to achieve a better global image segmentation is confirmed by simulation instructions. Hardware test platform is as follows:

Experimental environment:Window7, Intel(R) Core(TM) i7-3770; CPU:3.40GHz, RAM8.00G; 64-bit operating system; Preferences: $u=0.5, v=0.001 \times 255^{2}, \varepsilon=1$, time step $\Delta t=0.1$, the number of iterations is set to 50 times.

Algorithm flow is as follows:

(1) $\mathrm{t}=0$, the initialization $\phi$ :

$$
\phi(x, t=0)=\left\{\begin{array}{l}
-1, x \in \Omega_{0}-\rho \Omega_{0} \\
0, x \in \rho \Omega_{0} \\
1, x \in \Omega-\Omega_{0}
\end{array}\right.
$$

Wherein, $\Omega_{0}$ is a subset of the image area $\Omega, \rho \Omega_{0}$ is the sub-region $\Omega_{0}$ boundary.

(2) According to the equations (7)(12)(13) to update $\phi, C, b$, Energy function $F(\phi, C, b)$ is minimized.

(3) $\phi^{n+1}=\phi^{n}+\Delta t \bullet \Delta \phi^{n}$, wherein $\Delta t$ is iteration time step.

(4)Evolution (4) is to determine the equation of whether to stop, if not stopped, return to Step 2, otherwise ends.

Comparison of several image segmentation algorithm

The original CV algorithm, the improved algorithm LBF and the proposed new algorithm for Breast. tif, Noisy circle.tif, Heart-ct.bmp three images for simulation, respectively, in segmentation effect, Iterations times, time consuming comparison, the simulation results shown in Fig 1.

By comparing the results of the above-described segmentation, Fig.1(d),(g),(j)three images can be seen that, under the conditions the same initialization of the boundary, for Breast.tif image 
segmentation algorithm and modified the original CV the LBF model segmentation results are not good in this article segmentation algorithm, our algorithm can perform global image segmentation, while the other two algorithms spit a lot of sub- regions;(e),(h),(k) three figure, image Noisy-circle contain reflection noise, you can see the three algorithms are able to split out the target, but the original CV algorithm and improved model LBF more noise while split out as a target, but the algorithm is rarely segmented noise, can effectively resist interference noise, and effectively split the target, from (f),(i),(j)three figures, the three segmentation algorithms algorithm for image segmentation Heart-ct. bmp the best results.

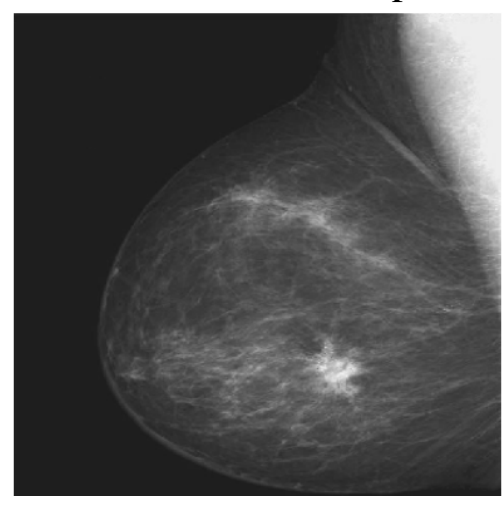

$\mathrm{a}$

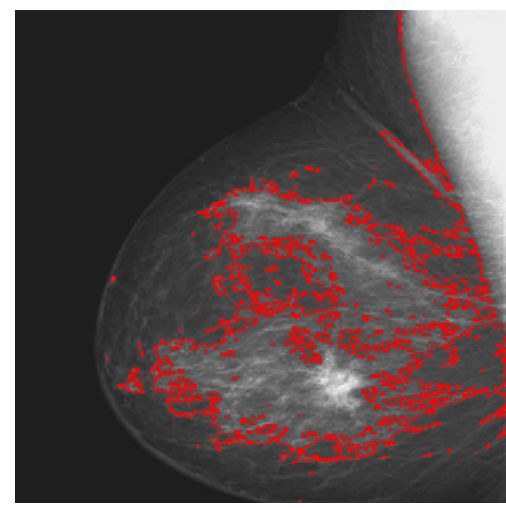

d

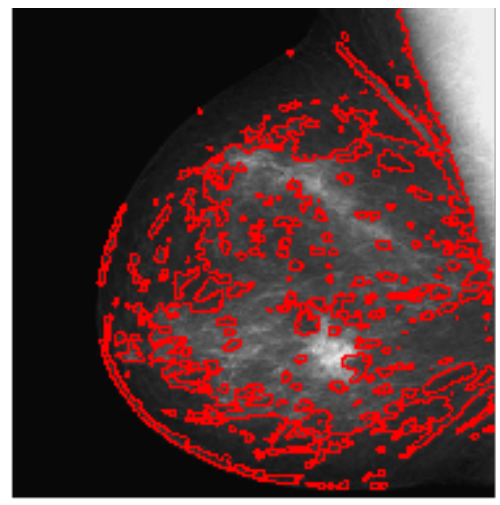

g

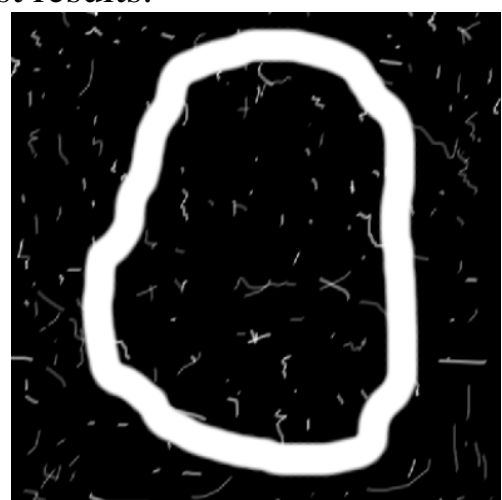

b

original image

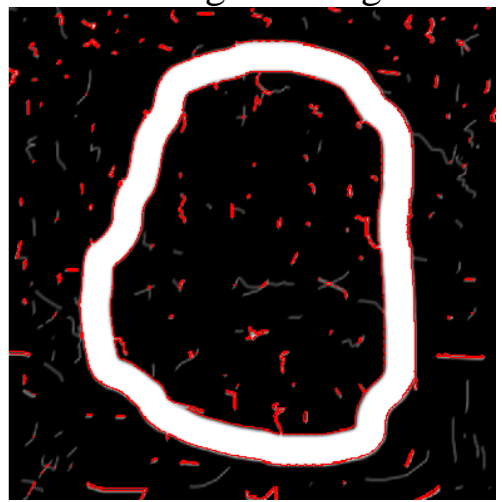

e

CV algorithm segme

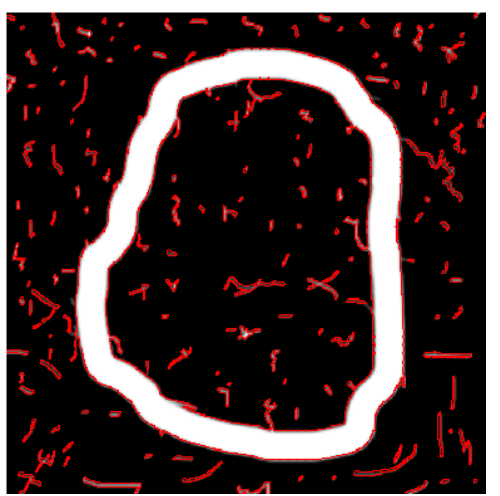

$\mathrm{h}$

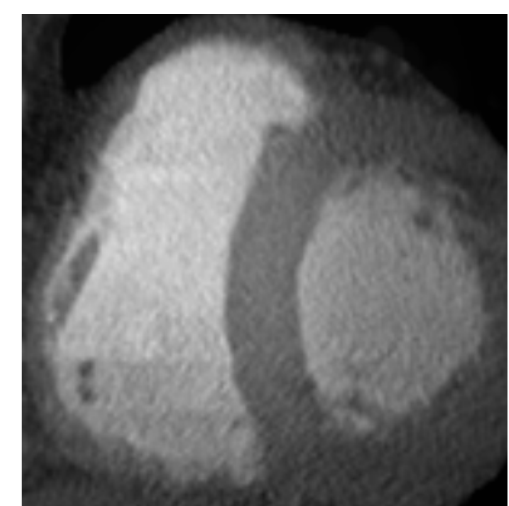

C

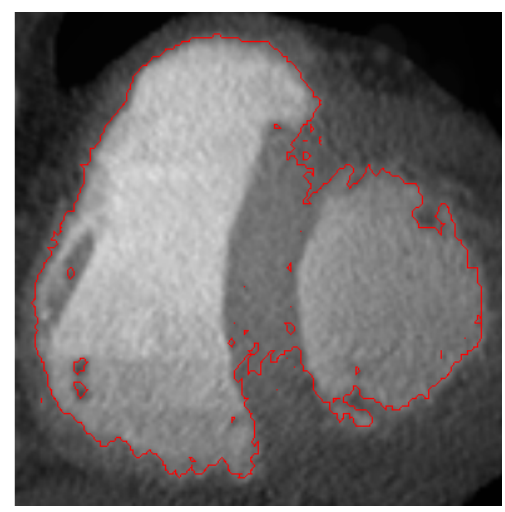

f

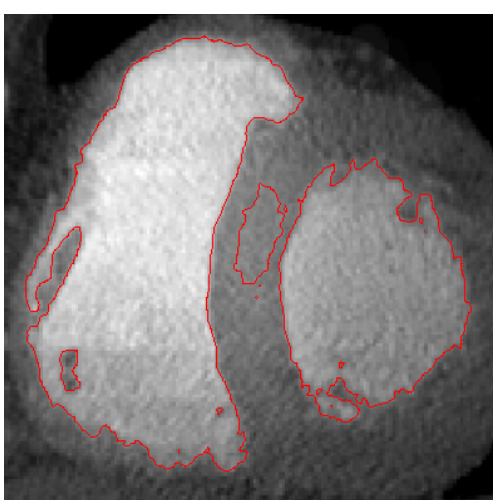

i

improved segmentation algorithm LBP map 


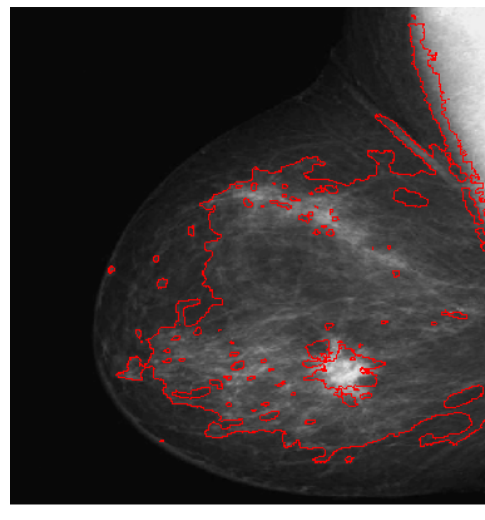

j

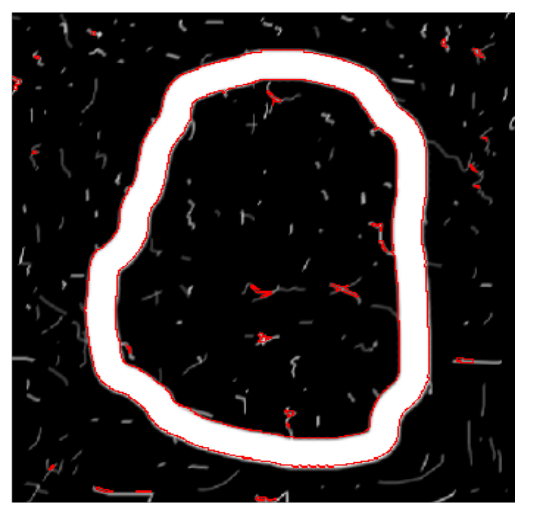

$\mathrm{k}$

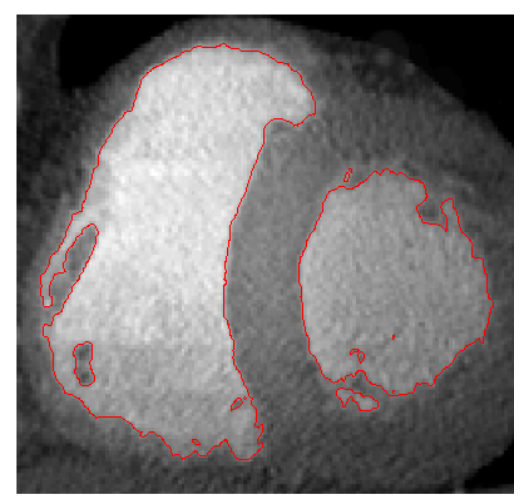

1

segmentation algorithm results map

Fig.1. Comparison of different algorithms for image segmentation results

(Figure a-c are original image; d-f for CV segmentation algorithm;Figure g-i LBF for improved

segmentation algorithm; Figure j-l algorithm for segmentation map)

Table 1 three image segmentation method and time-consuming iterations comparisons table

\begin{tabular}{|c|c|c|c|c|c|c|}
\hline & \multicolumn{2}{|c|}{ Breast.tif } & \multicolumn{2}{|c|}{ Noisy_circle.tif } & \multicolumn{2}{|c|}{ Heart_ct.bmp } \\
\hline & iterations & time & iterations & time & iterations & time \\
\hline $\mathrm{CV}$ & 1000 & $92.32 \mathrm{~s}$ & 1000 & $103.22 \mathrm{~s}$ & 1000 & 3.84 \\
\hline $\begin{array}{l}\text { Improved } \\
\text { algorithm }\end{array}$ & 10 & 22.9s & 10 & $27.30 \mathrm{~s}$ & 10 & 1.95 \\
\hline Proposed algorithm & 10 & $22.45 \mathrm{~s}$ & 10 & $25.79 \mathrm{~s}$ & 10 & 1.99 \\
\hline
\end{tabular}

\section{Summary}

We base this paper on the original image segmentation that is based on the level set by the inner and outer contours of local and global information adaptive fit to get a better overall segmentation, and segmentation of images also has good robustness. The new algorithm can be applied not only to the natural image segmentation can also be used in medical image segmentation and SAR images. In addition to the noise of the image, the image information will be subject to interference target occlusion,information loss and other factors. In this case, the image segmentation relies solely on the bottom of the image information of image segmentation, and can not get good segmentation results, thus how to solve complex background image segmentation problem with the use of a priori information and the bottom image remains unknown.

\section{References}

[1] T. Chan, L. Vese. Active contour without edges [J]. IEEE Transaction on Image Processing, 2001,10 (2) :266-277.

[2] R. Ronfard. Region-based strategies for active contour models[J]. International Journal of Computer Vision, 2002,46: 223-247.

[3] R. Malladi, J.A. Sethian, B.C. Vemuri. Shape modeling with front propagation: a level set approach[J]. IEEE Transaction on Pattern Analysis and Machine Intelligence, 1995,17:158-175.

[4] G. Aubert, P. Kornprobst. Mathematical Problems in Image Processing: Partial Differential Equations and the Calculus of Variations[M]. Springer, New York,2002.

[5] C. Li, C. Kao, J. Gore, Z. Ding. Minimization of region-scalable fitting energy for image segmentation[J]. IEEE Transactions on Image Processing, 2008, 17:1940-1949. 
[6] C. Li, C. Kao, J. Gore, et al. Minimization of region-scalable fitting energy for image segmentation [J]. IEEE Transactions on Image Processing, 2008,17:1940-1949.

[7] C.M. Li, C.Y. Xu, C.F. Gui, et al. Level set evolution without re-initialization: a new variational formulation[J]. IEEE Conference on Computer Vision and Pattern Recognition, San Diego, 2005, pp. 430-436.

[8] S. Osher, R. Fedkiw. Level Set Methods and Dynamic Implicit Surfaces[M]. Springer, New York, 2002.

[9] Chunming Li, Rui Huang, Zhaohua Ding, et al. A Level Set Method for Image Segmentation in the Presence of Intensity Inhomogeneities with Application to MRI [J]. IEEE Transactions on Image Processing, 2011,20(7):2007-2016.

[10] N. Paragios, R. Deriche. Geodesic active regions and level set methods for supervised texture segmentation[J]. International Journal of Computer Vision,2002, 46:223-247.

[11] HE Rui-ying. C-V model Containing edge information[J]. Computer Engineering and Applications,2012,48(18):181-186.

[12] CUI Wen-chao, WANG Yi, FAN Yang-yu, et al. Localized FCM Clustering with Spatial Information for Medical Image Segmentation and Bias Field Estimation[J]. International Journal of Biomedical Imaging,2013,6:1-8

[13] A. Vasilevskiy, K. Siddiqi. Flux-maximizing geometric flows[J]. IEEE Transaction on Pattern Analysis and Machine Intelligence, 2002, 24:1565-1578. 
Zhang Aili(), female(Chinese), Professor, the main research areas:

Li Sijia (Jan.1994-), female(Chinese), the main research areas: digital image processing;

Liu Tuanning (Jul.1991-), female(Chinese), the main research areas: digital signal processing;

Li Zhiyong (Jul.1967-), male(Chinese), associate professor, the main research areas: digital image processing;

Zhang Yu (1964-), male(Chinese), Professor, the main research areas: Communications and Networks.

Contact:

\begin{tabular}{|c|c|c|c|l|c|c|}
\hline $\begin{array}{c}\text { First } \\
\text { author }\end{array}$ & $\begin{array}{c}\text { Other } \\
\text { authors }\end{array}$ & Title & Mailing Address & $\begin{array}{c}\text { Zip } \\
\text { Code }\end{array}$ & $\begin{array}{c}\text { First } \\
\text { author's } \\
\text { phone and } \\
\text { e-mail }\end{array}$ & $\begin{array}{c}\text { Instructor's/ } \\
\text { other authors' } \\
\text { phone and } \\
\text { email }\end{array}$ \\
\hline Zhang & $\begin{array}{c}\text { Li Sijia, } \\
\text { Liu } \\
\text { Tuanning, } \\
\text { Li } \\
\text { Zhiyong, } \\
\text { Zhang Yu }\end{array}$ & $\begin{array}{c}\text { An improved } \\
\text { adaptive image } \\
\text { segmentation } \\
\text { method }\end{array}$ & $\begin{array}{c}\text { Xinxiang City } \\
\text { Construction Road } \\
\text { 46 South Normal } \\
\text { University } \\
\text { Computer and } \\
\text { Information } \\
\text { Engineer College, } \\
\text { Henan Province }\end{array}$ & 453007 & $\begin{array}{l}\text { 1590387128 } \\
6 \text { zal5148@1 }\end{array}$ & $\begin{array}{c}\text { Li Zhiyong } \\
\text { Zhiyongli88 } \\
\text { @sohu.com }\end{array}$ \\
\hline
\end{tabular}

